\title{
Measurement of $\psi(3770)$ parameters with KEDR detector at VEPP-4M.
}

\section{Korneliy Yu. Todyshev for the KEDR Collaboration*}

Budker Institute of Nuclear Physics, Novosibirsk, Russia

E-mail: todyshev@inp.nsk.su

We present a measurement of the mass and total width of the $\psi(3770)$ meson. Results were obtained using data collected with the KEDR detector at the electron-positron accelerator complex VEPP-4M. We suggest a consistent way to describe the $\psi(3770)$ lineshape which includes interference between resonant and non-resonant $D \bar{D}$ production. The results are :

$$
\begin{aligned}
M_{\psi(3770)} & =3778.0_{-1.5}^{+1.6}{ }_{-0.7}^{+0.6} \mathrm{MeV} \\
\Gamma_{\psi(3770)} & =22.4_{-3.4}^{+3.9}{ }_{-0.5}^{+0.6} \mathrm{MeV}
\end{aligned}
$$

35th International Conference of High Energy Physics - ICHEP2010,

July 22-28, 2010

Paris France

*Speaker. 


\section{Experiment description}

In 2004-2006 three scans in the $\psi(2 S)-\psi(3770)$ energy region were carried out with an integrated luminosity of $2.7 \mathrm{pb}^{-1}$. The data acquisition scenario for $\psi(2 S)$ is similar to that described in [1]. For $\psi(3770)$ the data sample was collected at 11 and 24 points of center-of-mass energy in the interval 3.67-3.92 GeV in 2004 and 2006, respectively. The visible cross section of $e^{+} e^{-} \rightarrow$ hadrons together with the results of the fits is shown in Fig. 1. At the points near the $\psi(3770)$ peak the energy calibrations using the resonant depolarization method (RDM) were performed before and after data accumulation. The RDM energy calibration was not required off the peak. Routine monitoring of the beam energy was performed using the Compton backscattering method [2]. The accuracy of the energy interpolation varies from 10 to $30 \mathrm{keV}$ during the whole experiment.

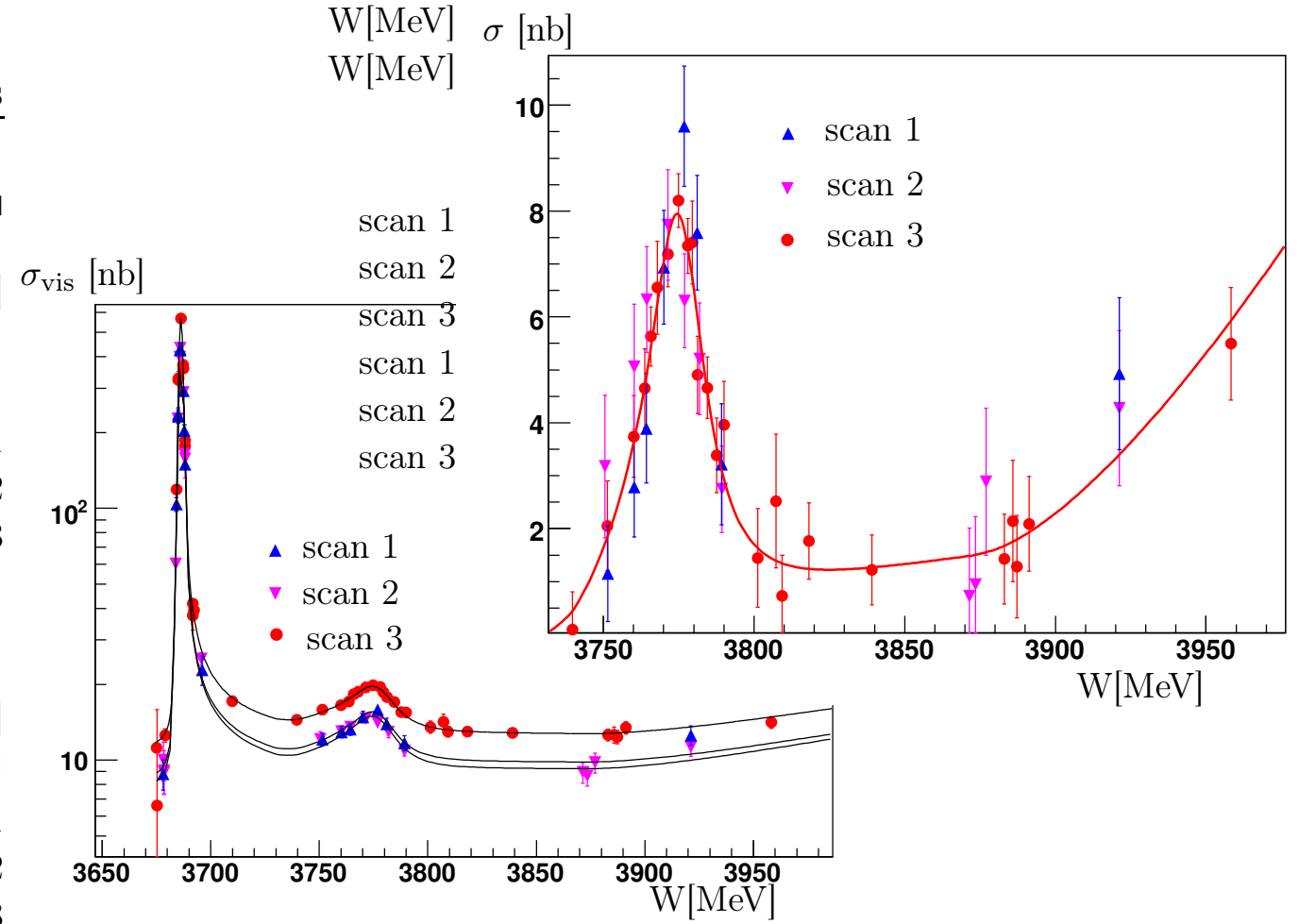

Figure 1: Visible cross section of $e^{+} e^{-} \rightarrow$ hadrons vs. CM energy for three scans (detection efficiencies are different) and cross section of $e^{+} e^{-} \rightarrow$ hadrons after light quark background subtraction. The lines are the result of a simultaneous fit.

\section{The multihadron cross section in the vicinity of $\psi(3770)$}

The non-relativistic $p$-wave Breit-Wigner with energy-dependent total width was used in early experiments $[3,4,5]$ to study inclusive cross section of the $\psi(3770)$ resonance. The non-resonant $D \bar{D}$ cross section was taken to be proportional to the sum of cubes of $\mathrm{D}$ meson momenta. Determination of the $\psi(3770)$ parameters in our analysis is based on the vector dominance model. 
The model systematic errors have been examined by comparing results from different resonance descriptions including interference with non-resonant $D \bar{D}$ production and form factor.

\section{Acknowledgments}

We greatly appreciate the efforts of our VEPP-4M colleagues to provide good operation of the accelerator complex and the staff of experimental laboratories for the permanent support in preparing and performing this experiment.

\section{References}

[1] V.M. Aulchenko et al., Phys. Lett. B 573, 63 (2003).

[2] N.Yu. Muchnoi, S.A. Nikitin, V.N. Zhilich, in proceedings of EPAC-2006 (2006),Edinburgh, Scotland, 26-30 Jun 2006, pp. 1181-1183.

[3] P. A. Rapidis et al., Phys. Rev. Lett. 39 (1977) 526.

[4] W. Bacino et al., Phys. Rev. Lett. 40 (1978) 671.

[5] R. H. Schindler et al., Phys. Rev. D21 (1980) 2716. 